\title{
Osteoartrose costovertebral: Diagnóstico diferencial raro de dorsalgia no paciente jovem. Relato de caso*
}

\section{Costovertebral Osteoarthrosis: Rare Differential Diagnosis of Back pain in young patients. Case Report}

\author{
João Ribeiro Afonso ${ }^{1}$ Diogo Soares ${ }^{1}$ Daniel Brás Lopes ${ }^{1}$ Rui Milheiro Matos ${ }^{1}$ Rui Peixoto Pinto ${ }^{1}$ \\ ${ }^{1}$ Departamento de Ortopedia e Traumatologia, Hospital Santa Maria, \\ Porto, Portugal \\ Endereço para correspondência João Ribeiro Afonso, MD, \\ Rua de Camões 906, 4049-025, Porto, Portugal \\ (e-mail: jmafonso89@gmail.com).
}

Rev Bras Ortop 2022;57(2):345-347.

\section{Resumo \\ Palavras-chave \\ - osteoartrite \\ - vértebras lombares \\ - vértebras torácicas}

O diagnóstico diferencial de dorsalgia revela-se um desafio pela proximidade da coluna dorsal a órgãos vitais assim como por sua anatomia única, inervação e articulação com as costelas. Os padrões de dor referida visceral obrigam, na maioria das vezes, a extensivos exames complementares de diagnóstico de forma a excluir condições graves. A osteoartrose da articulação costovertebral é um diagnóstico pouco reconhecido, e habitualmente é somente considerado quando a fonte de dor continua sem explicação após extensa investigação. Os autores apresentam o caso de um homem de 40 anos de idade com dor dorsal incapacitante devido a osteoartrose costovertebral isolada. A sintomatologia foi controlada após a injeção de metilprednisolona guiada por tomografia computadorizada. Este caso clínico tem como objetivo descrever a apresentação clínica de uma entidade rara que deverá ser considerada no diagnóstico diferencial de dorsalgia.

The differential diagnosis of dorsal thoracic pain can be a challenge due to the proximity of the dorsal column to vital organs as well as to its unique anatomy, innervation, and rib joint. The patterns of referred visceral pain require, in most cases, extensive complementary diagnostic tests in order to exclude severe conditions. Referred pain patterns often result in numerous and expensive visceral workups in order to exclude serious conditions, and costovertebral joint osteoarthritis is usually only considered when the origin of the pain remains unexplained. The authors present the case of a 40-year-old man with disabling dorsal pain due to isolated costovertebral osteoarthrosis. The symptomatology was controlled after injection of methylprednisolone guided by computed tomography. This clinical case aims to describe the clinical presentation of a rare entity that should be considered in the differential diagnosis of back pain.

Trabalho desenvolvido no Departamento de Ortopedia e Trau-

matologia, Hospital Santa Maria, Porto, Portugal.

recebido

13 de Setembro de 2020

aceito

09 de Outubro de 2020

published online

Março 31, 2021
DOI https://doi.org/

10.1055/s-0040-1722587. ISSN 0102-3616. (c) 2021. Sociedade Brasileira de Ortopedia e Traumatologia. All rights reserved.

This is an open access article published by Thieme under the terms of the Creative Commons Attribution-NonDerivative-NonCommercial-License, permitting copying and reproduction so long as the original work is given appropriate credit. Contents may not be used for commercial purposes, or adapted, remixed, transformed or built upon. (https://creativecommons.org/ licenses/by-nc-nd/4.0/)

Thieme Revinter Publicações Ltda., Rua do Matoso 170, Rio de Janeiro, RJ, CEP 20270-135, Brazil 


\section{Introdução}

A sintomatologia álgica com origem na coluna vertebral é uma queixa prevalente, sendo responsável por consideráveis custos diretos, inerentes à prestação de cuidados de saúde, $\mathrm{e}$ indiretos, devidos ao absentismo e perda de produtividade. O seu esclarecimento etiológico pode impor-se como um verdadeiro desafio diagnóstico dada a sua proximidade a órgãos vitais e ao fenômeno de dor referida que resulta da convergência neuronal aferente de fibras viscerais e somáticas ao nível da coluna dorsal. ${ }^{1,2}$ Isso explica o amplo espectro de diagnósticos diferenciais de dorsalgia, incluindo hérnia discal, fratura vertebral, alterações degenerativas facetárias, neuralgia intercostal, fraturas de costelas, e ainda patologias do foro não ortopédico, nomeadamente, cardíacas, renais, pulmonares e abdominais. ${ }^{3}$

Apesar de a osteoartrose (OA) da articulação costovertebral ser um achado degenerativo comum em idade geriátrica, ${ }^{4}$ a sua ocorrência isolada no paciente jovem é rara, sendo uma condição pouco reconhecida na origem de dor dorsal. ${ }^{5}$ Os autores apresentam o caso de um paciente jovem com um quadro de dor dorsal, irradiada ao hemitórax esquerdo, com origem na OA de uma articulação costovertebral. Esse caso destaca a complexidade do diagnóstico de dor dorsal e visa sensibilizar os médicos para a existência desse diagnóstico, a fim de evitar exames complementares dispendiosos e muitas vezes desnecessários direcionados à exclusão de patologia cardíaca ou pulmonar.

\section{Descrição do caso}

Um paciente de 40 anos de idade, do gênero masculino, relatou dor torácica com 4 semanas de evolução, de aparecimento insidioso, irradiada para o tórax esquerdo. A dor motivou duas idas ao serviço de emergência, onde o paciente foi avaliado por um especialista em medicina interna e submetido à realização de eletrocardiograma, radiografia de tórax, ecocardiograma, ecografia abdominal e estudo analítico (hemograma, velocidade de sedimentação, e proteína $C$ reativa). Todos os exames foram negativos para patologia cardíaca, pulmonar ou abdominal, sendo a dor interpretada num contexto de contratura do músculo trapézio inferior. Foram-lhe prescritos relaxantes musculares, fisioterapia, e o paciente foi, posteriormente, encaminhado para avaliação na nossa instituição. Os sintomas tornaram-se progressivamente incapacitantes com dor excruciante durante os movimentos de torção do tronco, inspiração profunda ou tosse, limitando significativamente as atividades de vida diária e a qualidade do sono. 0 paciente negou qualquer história de trauma, febre, fadiga ou perda ponderal. Relatou episódios prévios de dor, de natureza semelhante à atual, mas de menor intensidade e autolimitados, passíveis de alívio sintomático com a toma de antiinflamatórios. Ao exame objetivo, não apresentou assimetrias ou massas torácicas. A palpação das apófises espinhosas torácicas, do ligamento interespinhoso e da junção costotransversa foi indolor; no entretanto, a compressão das costelas inferiores esquerdas desencadeou dor paravertebral. 0 exame neurológico foi normal.
O paciente foi submetido à ressonância magnética da coluna dorsal, que revelou hiposinal nas sequências ponderadas em T1 e hipersinal nas sequências ponderadas em T2 ao nível da articulação costovertebral esquerda de D10. A patologia discal intervertebral foi excluída. Devido à natureza incerta da lesão, foram solicitadas uma tomografia computadorizada (TC) dorsal e uma cintigrafia óssea. A TC revelou a presença de alterações degenerativas da articulação costovertebral de D10 ( - Figura 1), em concordância com um aumento focal da captação de tecnécio-99m (Tc-99m) na mesma localização (- Figura 2), sendo os resultados a favor do diagnóstico de OA costovertebral. O doseamento dos marcadores inflamatórios (velocidade de sedimentação e proteína $\mathrm{C}$ reativa), marcadores reumatológicos (fator reumatoide, anticorpo antinuclear, anticorpos antidsDNA), HLA-B27, marcadores víricos e hemoculturas foram todos negativos. Foi proposta a injeção intralesional de corticoide guiada por TC com intuito diagnóstico e terapêutico. A articulação costovertebral esquerda de D10 foi primeiro devidamente identificada pela injeção local de $1 \mathrm{mLde} \mathrm{lidocaína}$ (20 mg/mL), que resultou num alívio imediato da dor. A resposta positiva ao anestésico local permitiu-nos não só confirmar a localização da lesão, bem como a confirmação do diagnóstico. Posteriormente, foram administrados localmente $2 \mathrm{~mL}$ de metilprednisolona $(40 \mathrm{mg} / \mathrm{mL})$. 0 procedimento decorreu sem intercorrências.

Foi observado um alívio progressivo da dor ao longo dos dias seguintes, com resolução completa às 3 semanas após a injeção de corticoide. A recorrência da dor não foi observada nos últimos 2 anos.

\section{Discussão}

O papel da OA costovertebral na origem da dorsalgia é pouco representado na literatura. A elevada prevalência de alterações degenerativas costovertebrais em pacientes assintomáticos obriga a uma interpretação cautelosa dos achados imagiológicos, sendo essencial a existência de uma correlação clínicoimagiológica. Apesar de estas serem bastante comuns em idade geriátrica, ${ }^{4}$ a sua ocorrência em pacientes jovens sem doenças do foro reumático é rara. Clinicamente, possuem um espectro extremamente variável, podendo ser assintomáticas ou ter uma apresentação altamente debilitante, com os pacientes a referirem dor torácica posterior, que pode irradiar para o tórax ou ser sentida ao longo da costela respectiva. ${ }^{3}$ A dor pode ser exacerbada por manobras provocatórias, nomeadamente com a inspiração profunda, tosse, flexão ou rotação do tórax e compressão da costela correspondente.

A publicação mais relevante sobre este tema é da autoria de Sales et al., ${ }^{5}$ que apresentou uma série de cinco casos de OA costovertebral isolada (idade média de 40,6 anos) tratados favoravelmente com a realização de artroplastia de resseção da costela.

A validade diagnóstica da cintigrafia óssea em condições degenerativas permanece questionável. ${ }^{6}$ Verdoorn et al. ${ }^{7}$ avaliaram a correlação entre a captação aumentada de Tc-99m nas articulações costovertebrais, a presença de dor local e resposta favorável à injeção percutânea de anestésico e corticoide. Os autores verificaram que mais da metade dos casos com 


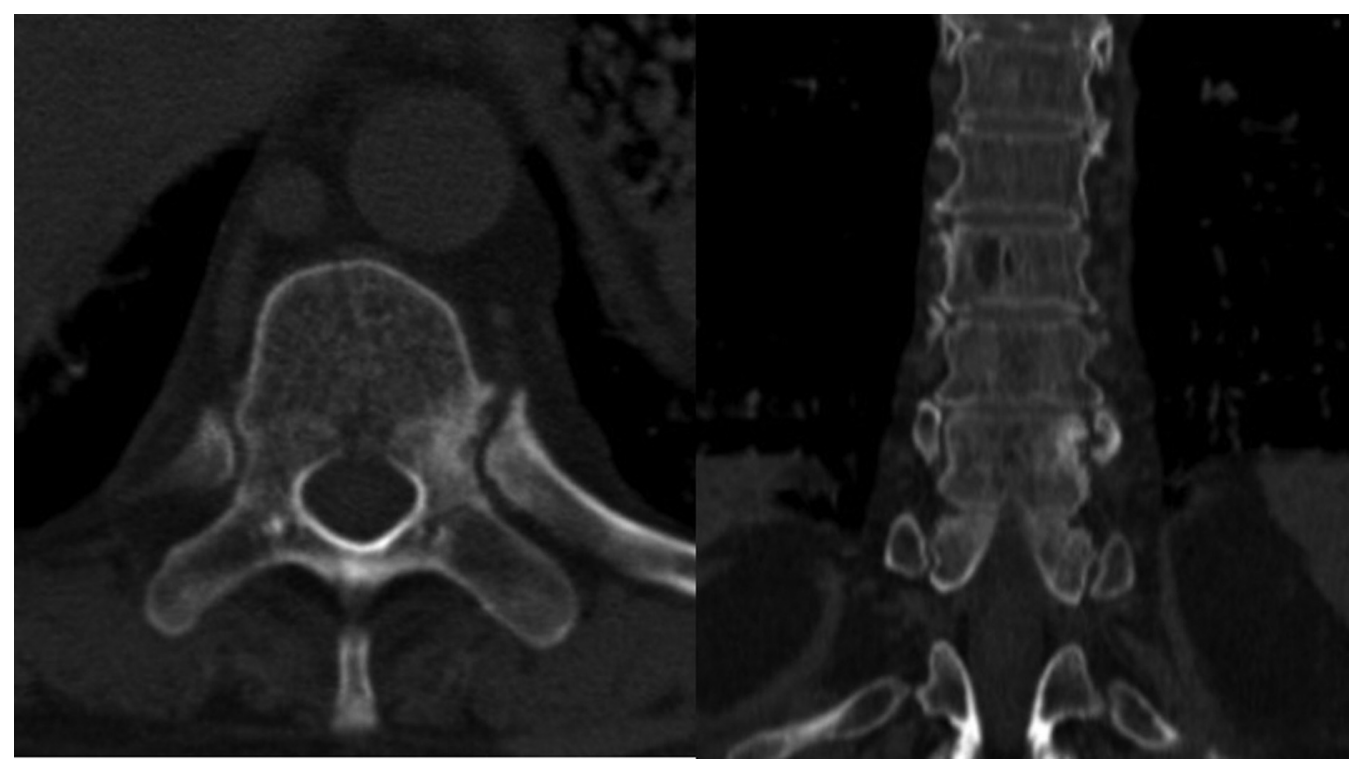

Fig. 1 Tomografia computadorizada. Corte axial (à esquerda) e coronal (à direita). A décima articulação costovertebral esquerda apresenta alterações degenerativas significativas, com esclerose nos níveis do corpo vertebral, do pedículo e da cabeça da costela.

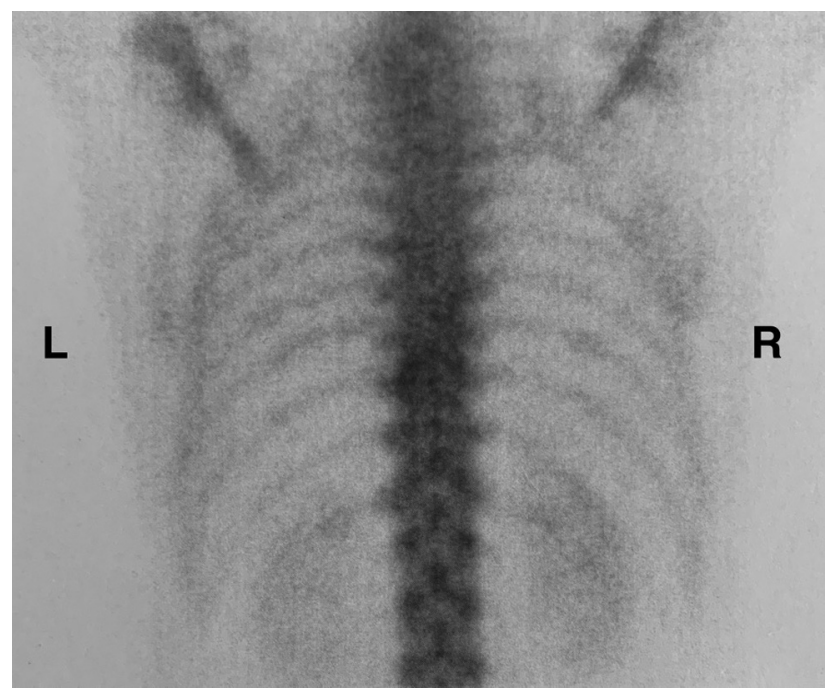

Fig. 2 Cintigrafia óssea marcada com Tc-99m. Hipercaptação isolada no nível da décima articulação costovertebral esquerda (L- lado esquerdo; R-lado direito).

hipercaptação de Tc-99m eram assintomáticos, não existindo uma correlação entre a presença de dor e a previsibilidade de resposta ao tratamento por injeção percutânea. Estes resultados são justificados pela possibilidade de existirem pontos dolorosos confundidores e pela presença de mais do que uma área de captação aumentada de Tc-99m, o que reduz a eficácia diagnóstica e a previsibilidade de resposta favorável ao tratamento com injeção percutânea de corticoide.

No nosso paciente, foi possível obter um alívio completo dos sintomas com a injeção de corticoide; no entanto, de acordo com os resultados relatados por Sales et al., ${ }^{5}$ é possível que haja uma recorrência dos sintomas a médio/ longo prazo, podendo a artroplastia de ressecção ser considerada com vista à obtenção de resultados duradouros.

\section{Conflito de Interesses}

Os autores declaram não haver conflito de interesses.

\section{Referências}

1 Saker E, Graham RA, Nicholas R, et al. Ligaments of the Costovertebral Joints including Biomechanics, Innervations, and Clinical Applications: A Comprehensive Review with Application to Approaches to the Thoracic Spine. Cureus 2016;8(11):e874

2 Young BA, Gill HE, Wainner RS, Flynn TW. Thoracic costotransverse joint pain patterns: a study in normal volunteers. BMC Musculoskelet Disord 2008;9:140

3 Fruth SJ. Differential diagnosis and treatment in a patient with posterior upper thoracic pain. Phys Ther 2006;86(02):254-268

4 Nathan H, Weinberg H, Robin GC, Aviad I. The Costovertebral Joints, Anatomical-Clinical Observations in Arthritis. Arthritis Rheum 1964;7:228-240

5 Sales JR, Beals RK, Hart RA. Osteoarthritis of the costovertebral joints: the results of resection arthroplasty. J Bone Joint Surg $\mathrm{Br}$ 2007;89(10):1336-1339

6 De Maeseneer M, Lenchik L, Everaert H, et al. Evaluation of lower back pain with bone scintigraphy and SPECT. Radiographics 1999; 19(04):901-912, discussion 912-914

7 Verdoorn JT, Lehman VT, Diehn FE, Maus TP. Increased 99mTc MDP activity in the costovertebral and costotransverse joints on SPECT-CT: is it predictive of associated back pain or response to percutaneous treatment? Diagn Interv Radiol 2015;21(04): 342-347 